\title{
The Innovation Canvas: An Experiential Tool to Stimulate Customer Discovery: An Abstract
}

\author{
Alizabeth M. Sánchez-López
}

\begin{abstract}
Despite all the progress in the field of entrepreneurship, there are still some unresolved issues. Understanding how opportunities are discovered and transformed is still hazy. The reality is that the learning paradigms employed by business schools have been based on behavioral approaches. Nonetheless behavioral approaches tend to overemphasize outcomes and routines and having one proper response to each stimulus which may prove useful for activities in organized environments that have clear goals, feedback, and reinforcement (e.g., established organizations at the execution stage) (Corbett 2005). Given that entrepreneurship is about searching and discovering opportunities, cognitive and situated learning may deem more appropriate. Moreover, recent claims posit that entrepreneurship has become more than a specific set of skills. Advocates argue that it is a more generalized method, similar to the scientific method. It's a way of reasoning and logic (Sarasvathy 2008).

Because of the above, this paper describes the Innovation Canvas, an experiential method that helps drive innovation and entrepreneurship (I\&E) and its components: discover, ideate, design, reframe, build, and test. Also, the paper presents examples on how to apply this framework (canvas) as well as techniques that could guide each stage. The underlying goal of this tool is to drive the opportunity identification process from a customer discovery lens while applying evidence-based entrepreneurship approaches.

The structure of the paper is as follows. First, learning theories are discussed in the light of the entrepreneurial process with an emphasis on the initial stages: search and discovery. Second, evidence-based entrepreneurship approaches derived from distinctive disciplines (e.g., design and software development) that attempt to provide guidance to the search and discovery phases of the entrepreneurial process are discussed. Third, the Innovation Canvas, an experiential learning tool that adopts and integrates from Customer Development, Lean Startup, and Design Thinking, is introduced. Finally, implications to theory and practice are discussed.
\end{abstract}

\footnotetext{
A.M. Sánchez-López $(\square)$

Universidad del Turabo, Gurabo, Puerto Rico

e-mail: asanchez63@suagm.edu
} 